Classification

Physics Abstracts

$61.30 \mathrm{G}-77.20$

\title{
Dielectric relaxation in liquid crystals exhibiting a reentrant phenomenon
}

\author{
C. Legrand, J. P. Parneix, A. Chapoton, Nguyen Huu Tinh $\left(^{*}\right)$ and C. Destrade (*) \\ Centre Hyperfréquences et Semiconducteurs ${ }^{+}$, Bât. P3, Université de Lille I, \\ 59655 Villeneuve d'Ascq Cedex, France \\ (*) Centre de Recherche Paul Pascal, 33405 Talence Cedex, France
}

(Reçu le 23 novembre 1983, révisé le 13 janvier 1984, accepté le 24 janvier 1984)

Résumé. - Nous présentons les résultats expérimentaux de la caractérisation diélectrique de mésomorphes présentant des phases rentrantes ou doublement rentrantes. La comparaison des énergies d'activation du mouvement de basculement moléculaire dans les différentes phases nous conduit aux conclusions suivantes : tique,

- l'énergie d'activation de la phase nématique rentrante est supérieure à celle de la phase néma-

- l'énergie d'activation de la phase smectique A rentrante est voisine de celle de la phase nématique rentrante.

A notre connaissance, cette étude est la première caractérisation diélectrique complète du phénomène doublement rentrant.

\begin{abstract}
This paper presents the experimental results of the dielectric characterization of mesomorphic compounds exhibiting reentrant or double reentrant phases. The comparison of activation energies in the flip-flop rotation of molecules in the different phases leads us to conclude that :

- the activation energy in the reentrant nematic phase is higher than that of the nematic phase,

- the activation energy in the reentrant smectic A phase is similar to that of the reentrant nematic phase.
\end{abstract}

To the best of our knowledge, this study presents the first complete dielectric characterization of the double reentrant phenomenon.

\section{Introduction.}

Reentrant phenomenon has been known for many years [1-4]. A dielectric study of the reentrant nematic phase and double reentrant phenomenon was made by Ratna et al. [5] and Benguigui et al. [6] respectively. We present here dielectric measurements on two pure compounds : 4cyanobenzoyloxy-4'-octylbenzoyloxy-p-phenylene (8 CBBP) 4-nitrobenzoyloxy-3-chlorobenzoate-p-decyloxyphenyl (10 ONBCBP) and a $60 \% 40 \%$ weight mixture of 4-decyloxybenzoate-6cyanonaphtyle (10 OBCN) and 4-nonyloxycinnamate-6-cyanonaphtyle (9 OCCN). The structural formulae and the phase sequences are given in table I. The different phases have been assigned

\footnotetext{
${ }^{+}$L. A. CNRS 287.
} 
Table I. - Chemical formulae and phase sequences of $8 \mathrm{CBBP}, 10 \mathrm{ONBCBP}$ and $10 \mathrm{OBCN} / 9$ OCCN mixture.

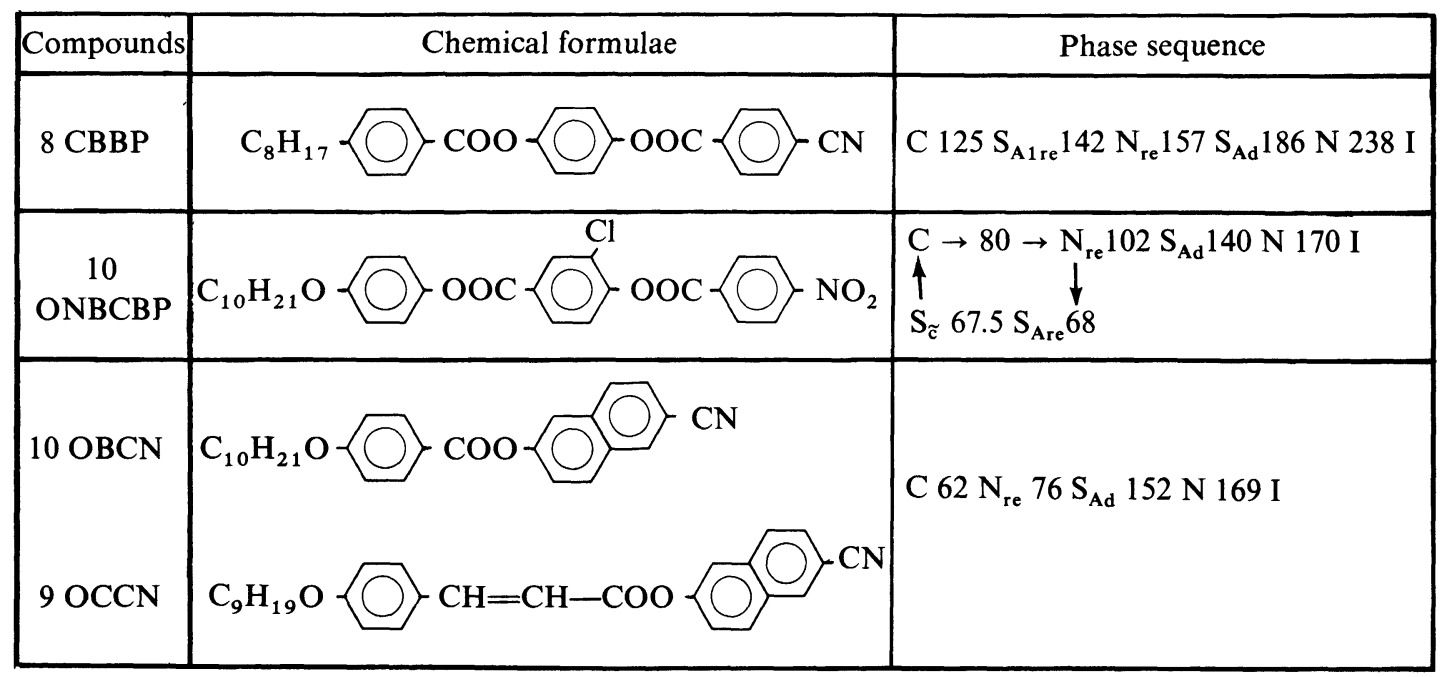

by their optical textures and checked by a contact method [7-9]. Note that 8 CBBP exhibits a reentrant nematic phase and a reentrant smectic $A$ phase. The temperature ranges of the various phases are large enough to allow a dielectric relaxation study except for the $S_{\text {Are }}\left(0.5^{\circ} \mathrm{C}\right)$ and $S_{\tilde{c}}$ phases of 10 ONBCBP. These phases are not very metastable and are difficult to manipulate (instant crystallization).

\section{Experimental conditions.}

The permittivities $\left(\varepsilon^{*}=\varepsilon^{\prime}-j \varepsilon^{\prime \prime}\right)$ are measured in each phase in the frequency range : $5 \mathrm{~Hz}$ $1 \mathrm{GHz}$. The basic devices are two impedance analysers HP 4192A (5 Hz-13 MHz) and HP $4191 \mathrm{~A}$ ( $1 \mathrm{MHz}-1 \mathrm{GHz}$ ) connected to a HP 9826 computer. They allow us to perform automatic measurements and give, in real time, the complex permittivity of the sample which can be plotted on a printer or a recorder. The measuring cell consists of two plane capacitors located at the end of a coaxial line [10]. This cell is usable for samples having high phase transition temperatures within a wide frequency range $(1 \mathrm{~Hz}$ to $1 \mathrm{GHz})$. Moreover a small sample volume is necessary $\left(<40 \mathrm{~mm}^{3}\right)$. In anisotropic phases, the permittivities $\varepsilon_{\|}^{*}$ and $\varepsilon_{\perp}^{*}$ are obtained when the measurement applied electric field is parallel or perpendicular to the optical axis respectively. A magnetic field of $1.2 \mathrm{~T}$ is used to orientate the sample optical axis. The accuracy of the temperature determination is better than $1 \%$ while the temperature is maintained constant within $\pm 0.1{ }^{\circ} \mathrm{C}$. The overall accuracy of the measured complex permittivities is better than $2 \%$ for $\varepsilon^{\prime}$ and $5 \%$ for $\varepsilon^{\prime \prime}$.

\section{Results.}

3.1 STATIC PERmitTivities. - The dielectric constant $\varepsilon_{\mathrm{s} \|}^{\prime} \varepsilon_{\mathrm{s} \perp}^{\prime}$, the static anisotropy $\Delta \varepsilon=$ $\varepsilon_{s \|}^{\prime}-\varepsilon_{s \perp}^{\prime}$ and $\bar{\varepsilon}=\left(\varepsilon_{s \|}^{\prime}+2 \varepsilon_{s \perp}^{\prime}\right) / 3$ as a function of temperature are given in figures $1,2,3$ for the investigated substances.

8 CBBP (Fig. 1) : The results show only slight variations of the dielectric constants and a positive anisotropy $(\sim 5)$ which increases with decreasing temperature. We did not note the maximum value of $\varepsilon_{\mathrm{s} \|}^{\prime}$ and $\varepsilon_{\mathrm{s} \perp}^{\prime}$ in the reentrant nematic phase as previously observed on a compound exhibiting double reentrant phenomenon [6]. 


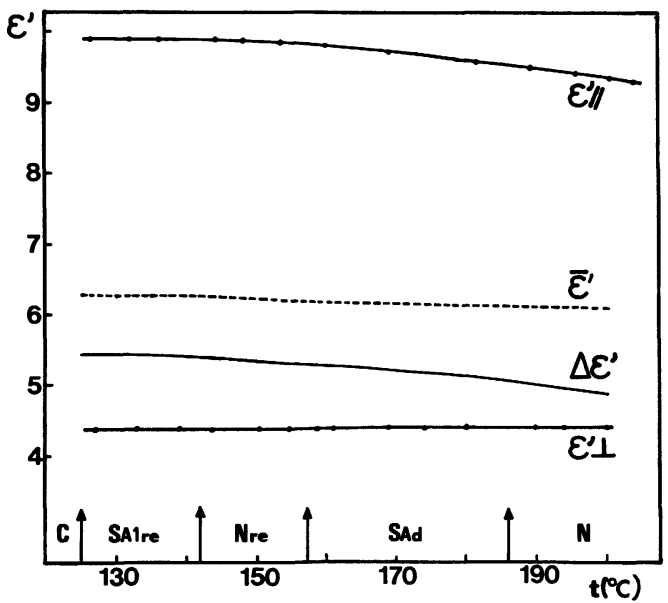

Fig. 1. - Static permittivities $\varepsilon_{\mathrm{s} \|}^{\prime}, \varepsilon_{\mathrm{s} \perp}^{\prime}, \bar{\varepsilon}$ and $\Delta \varepsilon$ as a function of temperature for $8 \mathrm{CBBP}$.

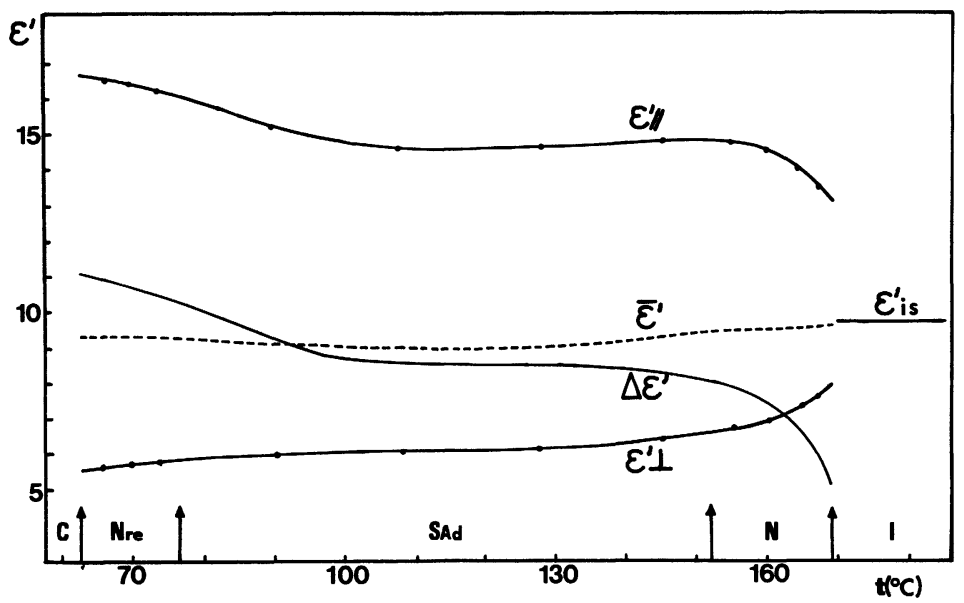

Fig. 2. - Static permittivities $\varepsilon_{\mathrm{s} \|}^{\prime}, \varepsilon_{\mathrm{s} \perp}^{\prime}, \bar{\varepsilon}$ and $\Delta \varepsilon$ as a function of temperature for $10 \mathrm{OBCN} / 9 \mathrm{OCCN}$ mixture.

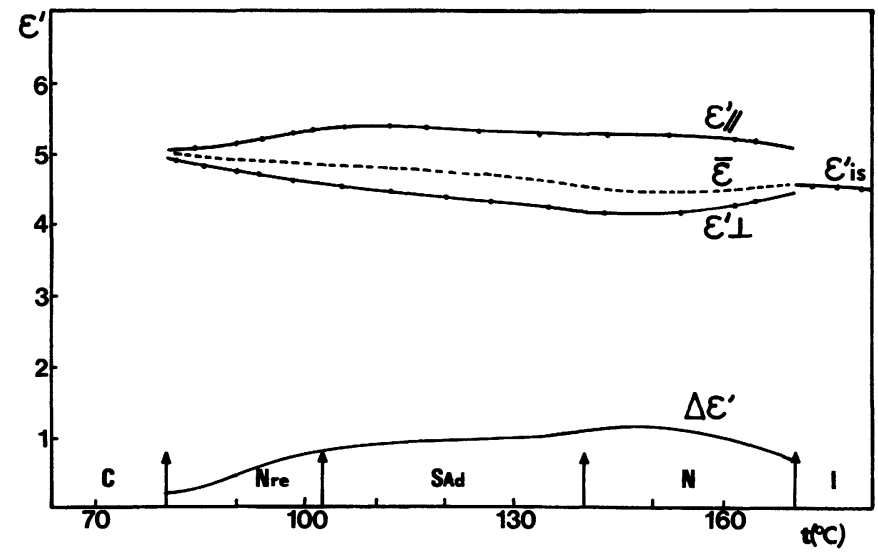

Fig. 3. - Static permittivities $\varepsilon_{s \|}^{\prime}, \varepsilon_{s \perp}^{\prime}, \bar{\varepsilon}$ and $\Delta \varepsilon$ as a function of temperature for 10 ONBCBP. 
9 OCCN/10 BCN (Fig. 2) : We found a high positive anisotropy ( 10) which increases with decreasing temperature. This increase is much larger near the reentrant nematic phase. As usual, $\bar{\varepsilon}$ is lower than the extrapolated isotropic value. In the nematic phase and in part of the smectic phase, $\bar{\varepsilon}$ decreases with decreasing temperatures and then increases for the lowest temperatures.

10 ONBCBP (Fig. 3) : For decreasing temperatures, the slighly positive anisotropy $(\sim 1)$ increases in the nematic phase then decreases in the smectic phase and more abruptly in the reentrant nematic phase. Such a behaviour was previously observed with a nematic compound of similar structure (alkoxy chain replaced by an alkyl chain and chlorate group replaced by a methyl group) [11] : the anisotropy sign of this compound changes.

3.2 Dynamic anisotropy. - The Cole and Cole diagrams obtained in the different phases for all the compounds are quite similar. As an example, we show in figures 4, 5, 6 those obtained for 10 ONBCBP in the isotropic phase and in the reentrant nematic phase.

In the isotropic phase (Fig. 4) and in the anisotropic phases when the electric field is perpendicular to the optical axis (Fig. 5), we observed domains which were distributed due to several mechanisms such as the rotation and libration of the molecules or intramolecular movements [12].

In the anisotropic phases when the electric field is parallel to the optical axis (Fig. 6), we observed two separate domains : the first corresponds to the flip-flop rotation of molecules around a transverse axis. Its relaxation frequency is lower than those corresponding to all other mechanisms [12].

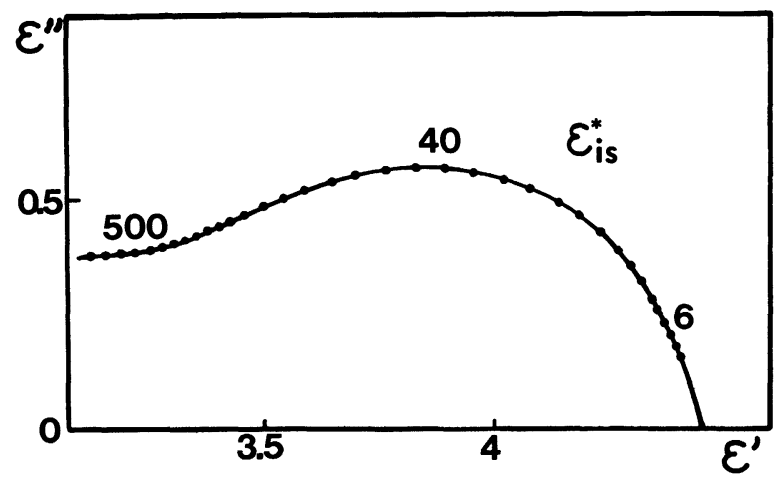

Fig. 4. - Cole and Cole diagram in the isotropic phase for $10 \mathrm{ONBCBP}(F$ in $\mathrm{MHz})$.

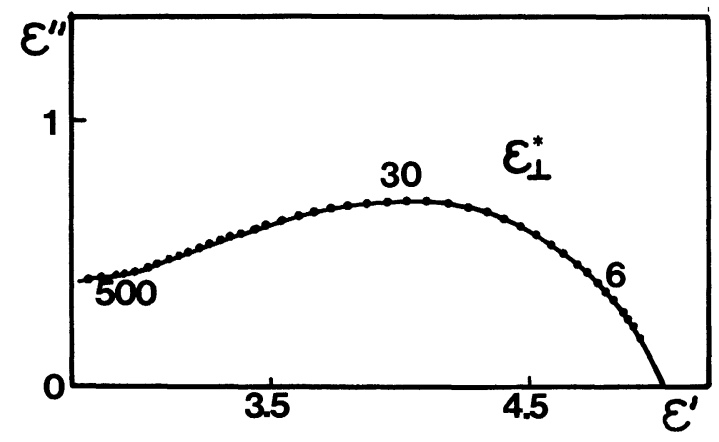

Fig. 5. - Cole and Cole diagram in the reentrant nematic phase when $\mathbf{E} \perp \mathbf{n}$ for $10 \mathrm{ONBCBP}(F$ in $\mathrm{MHz})$. 


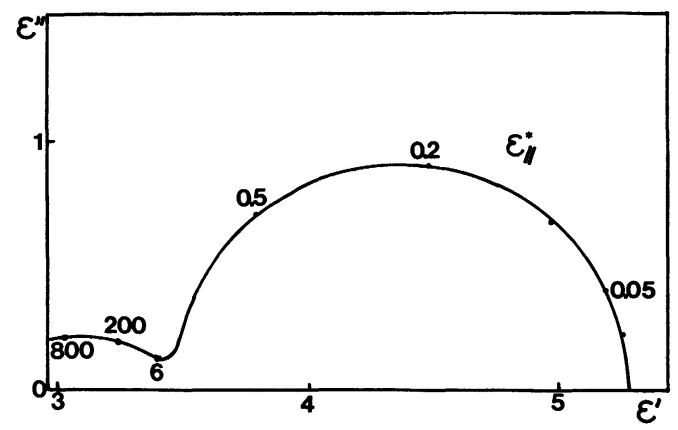

Fig. 6. - Cole and Cole diagram in the reentrant nematic phase when $\mathbf{E} / / \mathbf{n}$ for $10 \mathrm{ONBCBP}(F$ in $\mathrm{MHz})$.

The second one presents a small amplitude. It is connected to the transversal dipole moment $\mu_{\mathrm{t}}$ (rotation, libration) [12].

The variations of the flip-flop relaxation frequencies versus temperature are shown in figures 7 , 8 , 9. The different slopes give the activation energies of each phase. This leads us to the following observations :

- In all cases, the activation energy is higher in the nematic phase than in the smectic Ad phase. Similar results have been previously observed with compounds exhibiting reentrant nematic phase [5] or not [13].

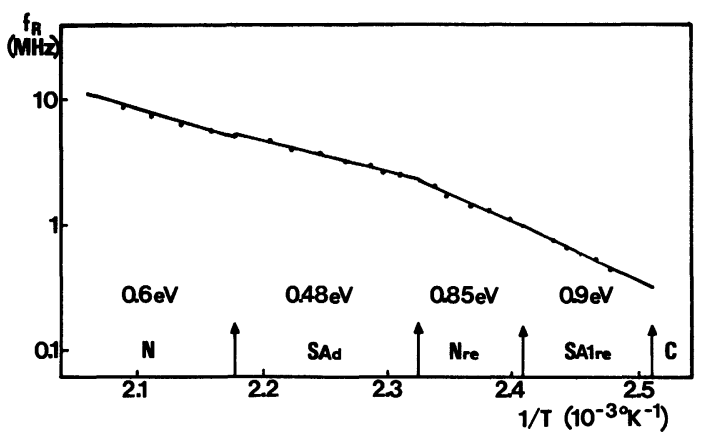

Fig. 7. - Relaxation frequencies versus inverse of temperature and activation energies for 8 CBBP.

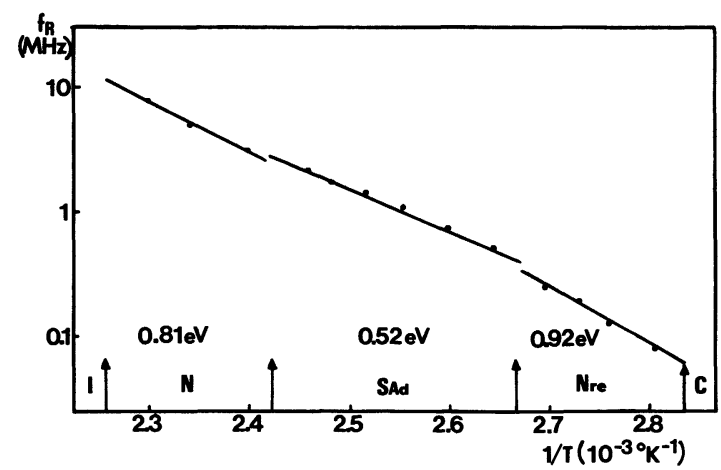

Fig. 8. - Relaxation frequencies versus inverse of temperature and activation energies for 10 ONBCBP. 


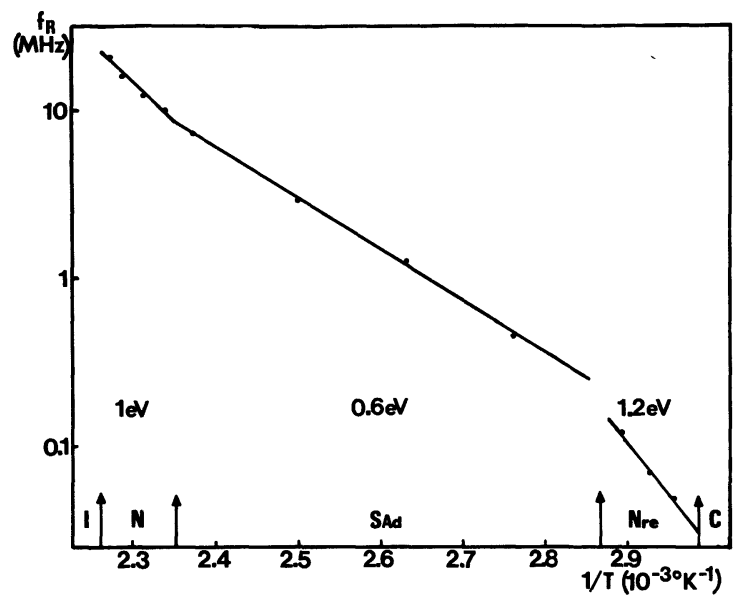

Fig. 9. - Relaxation frequencies versus inverse of temperature and activation energies for $10 \mathrm{OBCN} /$ 9 OCCN mixture.

- For all the substances, the activation energy is higher in the reentrant nematic phase than in the normal nematic phase. This is in agreement with the results of B. R. Ratna et al. [5].

- For 8 CBBP, we did not find the two relaxation frequencies in the reentrant nematic phase as did Benguigui et al. on T8 [6] and which they explained as being due to the existence of cybotatic groups of the two smectic phases. We obtained similar activation energies in the reentrant nematic phase and in the reentrant smectic Al phase. Activation energies in the nematic phase and in the smectic Ad phase of compounds exhibiting these two phases were found to be similar [13]. From these results, it would seem that the activation energy in the smectic A1 phase (reentrant or not) and the activation energy in the nematic phase which precedes it (when it exists) are similar, but further experiments would be necessary to prove this.

\section{References}

[1] Cladis, P. E., Phys. Rev. Lett. 35 (1975) 48.

[2] Cladis, P. E., Bogardus, R. K., Daniels, W. B., Taylor, G. N., Phys. Rev. Lett. 39 (1977) 720.

[3] Hardouin, F., Sigaud, G., Achard, M. F., Gasparoux, H., Phys. Lett. A 71 (1979) 347.

[4] Madhusudana, N. V., Sadashiva, B. K., Moodithaya, K. P. L., Current Sci. 48 (1979) 613.

[5] Ratna, B. R., Shashidhar, R., Rao, K. V., presented at the Int. Liq. Crystals Conf. Bangalore (1979).

[6] Benguigui, L., Hardouin, F., J. Physique Lett. 42 (1981) L-111.

[7] Nguyen Huu Tinh, Destrade, C., Nouveau J. Chim. 5 (1981) 337.

[8] Nguyen Huu Tinh, Destrade, C., To be published.

[9] NguYen Huu Tinh, Mol. Cryst. Liq. Cryst. 91 (1983) 285.

[10] Druon, C., Wacrenier, J. M., J. Phys. E. 16 (1983) 151.

[11] Madhusudana, N. V., Srikanta, B. S., Subramanya, M., Mol. Cryst. Liq. Cryst. 82 (1982) 25.

[12] Parneix, J. P., Chapoton, A., Mol. Cryst. Liq. Cryst. 78 (1981) 115.

[13] Druon, C., WaCrenier, J. M., Mol. Cryst. Liq. Cryst. 98 (1983) 201. 